\title{
Image Enhancement Algorithm Based on Dual Tree Complex Wavelet Transform
}

\author{
Chen Rong ${ }^{1, \text { a }}$ \\ ${ }^{1}$ Department of Physics, Nanchang Normal University, Nanchang, 330032, China \\ aemail: xinseachen@126.com
}

Keywords: Image; Enhancement; Algorithm; Dual Tree Complex Wavelet Transform

\begin{abstract}
Image processing is widely used in many fields,in image enhancement processing,traditional image enhancement method,such as histogram equalization, wavelet coefficient enhancement,and so on.Although these methods achieved good image enhancement performance,however,noise is amplified during image enhancement processing.In this paper,aiming at the problem,image enhancement algorithm based on dual tree complex wavelet transform(DT-CWT) is proposed.Firstly,image signal is decomposed by using DT-CWT,real tree and imaginary tree are obtained.And then the threshold processing of high frequency coefficients is performed.Finally,image signal is reconstructed by using DT-CWT.Experimental results show that the proposed method has better effect for image enhancement.
\end{abstract}

\section{Introduction}

In 1980s, the theory of wavelet analysis is introduced into the engineering application by DAUBECHIES and MALLAT.Wavelet transform can reflect local characteristics of signal,which is very important in the processing of weak random signal mixing in strong background noise.Although discrete wavelet transform is used widely,but there are still some disadvantages.A signal is sampled at intervals which will lose some information.Discrete wavelet transform can produce frequency aliasing in the process of decomposition and reconstruction.It will make the wavelet decomposition coefficient can not accurately reflect the state information.So that the feature extraction effect is affected.In order to overcome the disadvantages, a new method is put forward.Dual tree complex wavelet theory is put forward for the first time by N.G.Kingsbury who is Cambridge University scholar in 1998. The theory of dual tree complex wavelet algotithm is further development by Selesnick et. DT-CWT has been used in signal denoising,image denoising and image texture feature extraction,and so on. Which has been successfully used in the field of image processing $^{[1]}$.

\section{The Principles of Dual-Tree Complex Wavelet Transform}

DT-CWT is wavelet transform which using two parallel discrete wavelet (DWT) to complete.DT-CWT is a kind of complex wavelet transform which can be separated.The first road transform get real part,imaginary part is decomposed into second road transformation ${ }^{[2-3]}$.In the decomposition and reconstruction process of signal,the sampling position of imaginary tree is always in the middle of real tree,so that it can effectively use wavelet decomposition coefficients of real part and imaginary part to relize information complementary and approximate shift invariance of real tree and imaginary tree,to reduce loss of useful information.In the decomposition process of DT-CWT which use wavelet coefficient dichotomy to reduce redundant computation,to improve the computational speed.The decomposition and reconstruction process of DT-CWT is as shown in Figure 1.Among them, $h_{0}$ is a low pass filter for real tree, $h_{1}$ is a high pass filter for real tree. $g_{0}$ is a low pass filter for imaginary tree, ${ }^{g_{1}}$ is a high pass filter for imaginary tree, $\downarrow 2$ represent interval sampling. 


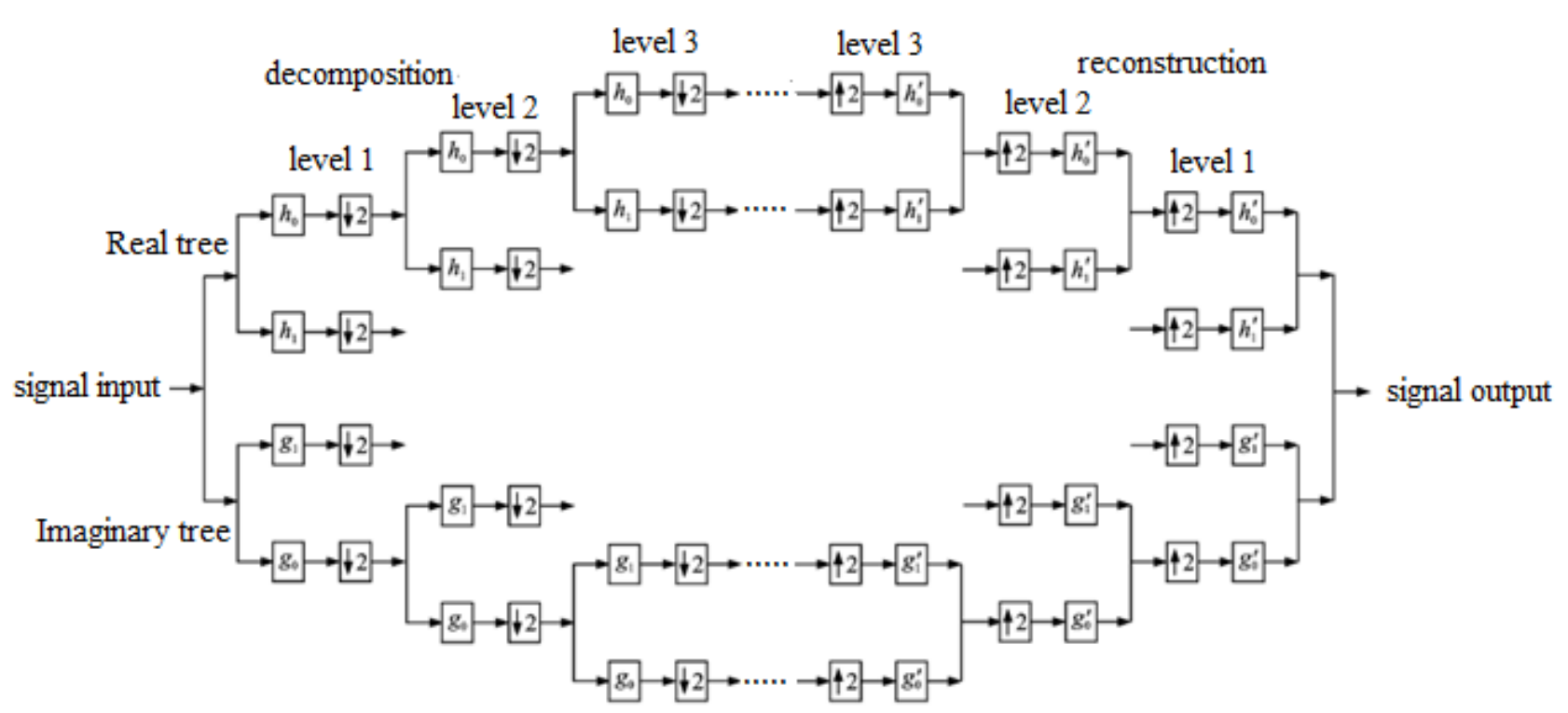

Fig.1.The decomposition and reconstruction of DT-CWT

\section{The Main Idea of Dual Tree Complex Wavelet Transform}

The first layer of wavelet decomposition,real tree and imaginary tree between the delay of filters should be maintained at a sampling interval,so that imaginary tree can be ensured to binary sample missing sampling values of real tree.Each layer wavelet decomposition after first layer,phase frequency response of filters should be existed half a sampling period of group delay,and amplitude frequency response of filters equal exists.In order to guarantee linear phase,Kingsbury requires that length of one tree filter is odd,and the other is even for real tree and imaginary tree ${ }^{[4-5]}$.The basic steps of DT-CWT is:(a) DT-CWT decomposition.The layer of dual tree complex wavelet decomposition must be set,obtained a group of coefficients for signal decomposition.(b)high frequency coefficients are processed by threshold.To select the appropriate threshold,high frequency coefficients of each layer are processed,then high frequency coefficients are obtained.(c)DT-CWT reconstruction.The high frequency coefficients and low frequency coefficients after threshold processing by DT-CWT reconstruction,get signal after denoising.

The basic information of signal is contained in the low frequency part of the wavelet coefficients.The noise is contained in the high frequency part of it.At each decomposition scale,a suitable threshold must be set.the value of wavelet coefficient is smaller than the threshold,which is set to zero.And the value of the wavelet coefficient is higher than the threshold,it remain unchanged.After the processing,signal can retain useful information to achieve the purpose of removing noise.

\section{Experimental Results}

Since some images are 2D,so DT-CWT needs to be extended to two-dimensional DT-CWT,two-dimensional discrete wavelet transform can be used in two trees.Both real and imaginary parts of two-dimensional DT-CWT can describe 6 main directions $\left( \pm 15^{\circ}, \pm 45^{\circ}, \pm 75^{\circ}\right)$, which can reflect the change of image in different scales, and it is suitable to extract the local features of image.In other words,after decomposition of each level,2 low frequency sub bands are obtained at the same time,and 6 high frequency sub bands are obtained.Each layer has 6 different directional information, which can further improve the accuracy of decomposition and synthesis of image,so as to extract information of original image from 6 different directions.Figure 2 shows the simulation result for decomposition and reconstruction based on DT-CWT. 


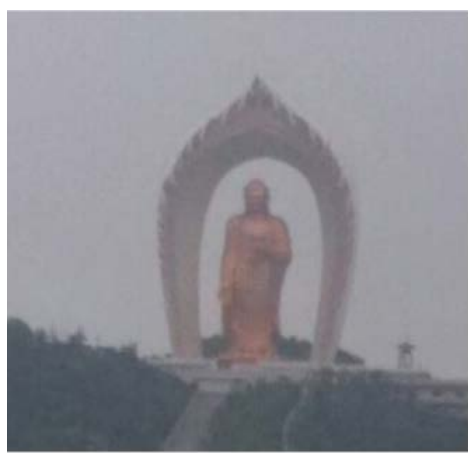

(a)
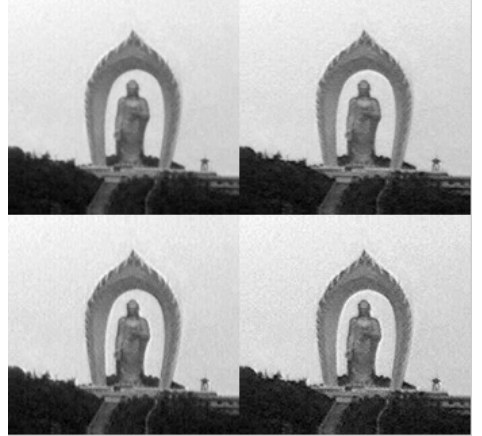

(d)

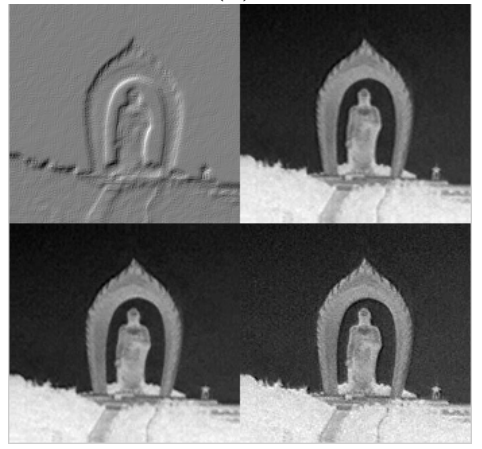

(g)

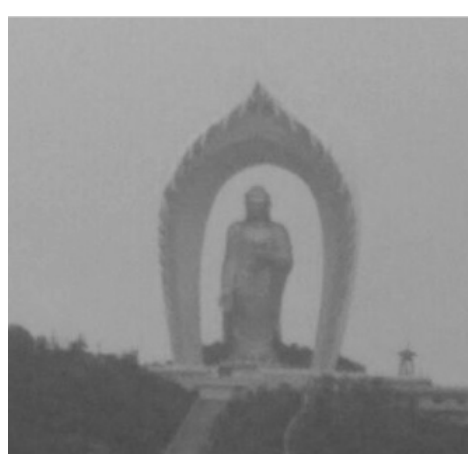

(b)

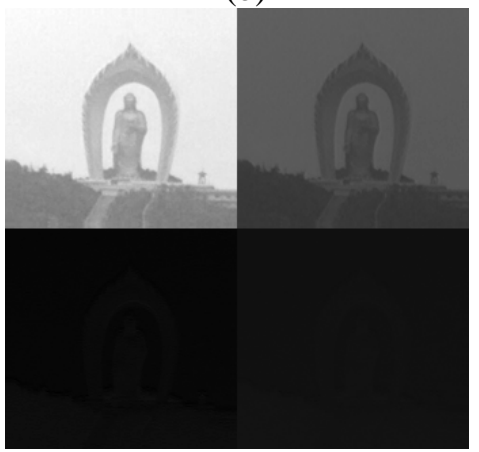

(e)

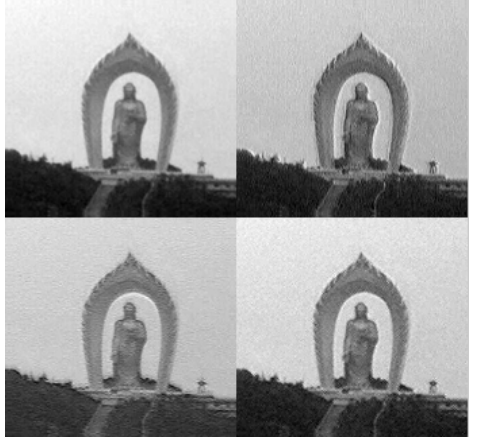

(h)

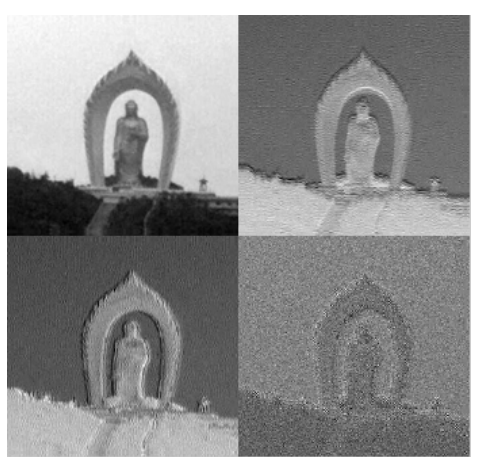

(c)

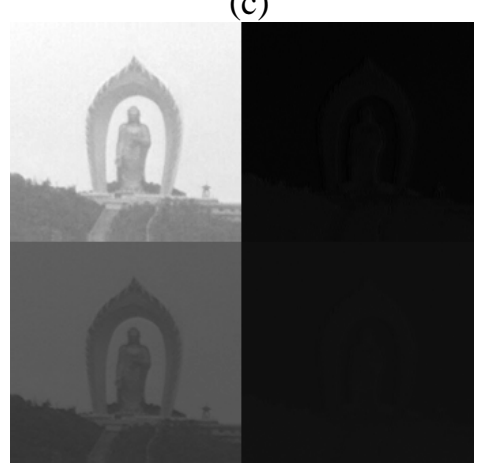

(f)

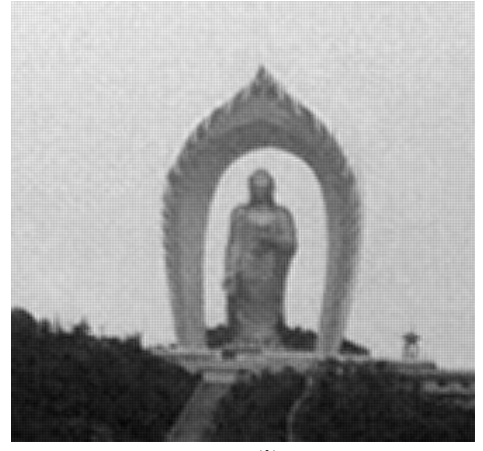

(i)

(a)Orgin image RGB(b)Orgin image Gray (c)The image is transformed by $\{\mathrm{h} 0, \mathrm{~h} 1\}, \mathrm{A}$ approximation coefficients, $\mathrm{H}$ horizontal detail coefficients, $\mathrm{V}$ vertical detail coefficients, $\mathrm{D}$ diagonal detail coefficients(d)The image is transformed by $\{\mathrm{g} 0, \mathrm{~g} 1\}$, A approximation coefficients, $\mathrm{H}$ horizontal detail coefficients, $V$ vertical detail coefficients, $D$ diagonal detail coefficients(e)First use of the filter $\{\mathrm{g} 0, \mathrm{~g} 1\}$ to do the line transform,and then the filter $\{\mathrm{h} 0, \mathrm{~h} 1\}$ do column transformation (f)First use of the filter $\{\mathrm{h} 0, \mathrm{~h} 1\}$ to do the line transform, and then the filter $\{\mathrm{g} 0$, g1 $\}$ do column transformation(g)From top to bottom,from left to right,the decomposition of real tree has 4 sub bands, one is low frequency band,others are high frequency sub band $\left(-15^{\circ},-45^{\circ},-75^{\circ}\right)$.(h)From top to bottom,from left to right,decomposition of imaginary tree has 4 sub bands,one is low frequency band, others are high frequency sub band $\left(+15^{\circ},+45^{\circ},+75^{\circ}\right)$.(i)Reconstruction image.

Fig.2. The simulation result of DT-CWT

\section{Conclusion}

DT-CWT has some advantages including shift invariance and directional selection.The decomposition of DT-CWT get low frequency components and high frequency components.According to the characteristics of low frequency sub-images concentrating the majority energy of source image and determining image contour.Denoising algorithm based on DT-CWT also has a great enhancement to signal.And then the threshold processing of high frequency coefficients is performed.Finally,signal is reconstructed by using DT-CWT.Experimental results show that many features of image such as edges and textures can be preserved well after filtering via the proposed algorithm. The algorithm enriches detailed information of images besides 
keeping original images information.The proposed method has better effect for image enhancement.

\section{Acknowledgement}

In this paper,the research was sponsored by key discipline of Nanchang Normal University,China (Project No.NSXK20141001) and reform in education of Nanchang Normal University,China(Project No.JGKT-14-18).Also it was sponsored by Scientific Research of Nanchang Normal University(Project No.15KJYB41).

\section{References}

[1]Wang Fang,Ji Zhong,Peng Chenglin.Research on ECG signal denoising based on dual-tree complex wavelet transform[J].Chinese Journal of Scientific Instrument,2013,34(5):1160-1166

[2]Wang Shimin,Ye Jihua,Deng Tao,Wang Mingwen. Two-dimensional dual-tree complex wavelet transform uncertainty weighted fusion in face recognition[J].Journal of Image and Graphics,17(8):995-1001

[3]Ren Zhiying,Gao Chenghui,Lin Jianxing,Shen Ding.Evaluation Method of Three-dimensional Rough Surface Based on Dual-tree Complex Wavelet Transform Methods[J].Journal of Mechanics in Engineering,2014,25(13):1795-1799

[4]Kingsbury N G.The dual-tree complex wavelet transform:a new technique for shift invariance and directional filters[C]//Proceedings of the 8th IEEE Digital Signal Processing Workshop,Bryce Canyon, USA, 1998. Piscataway, NJ, USA: IEEE,1998: 86-89

[5]Chakraborty S,Bhattacharya I,Chatterjee A.Apalmprint based biometric authentication system using dual tree complexwavelet transform[J].Measurement,2013,46(10):4179-4188 